\title{
Plane Symmetric Self-Gravitating Fluids with Pressure Equal to Energy Density
}

\author{
Romualdo Tabensky \\ Physics Department, University of California, Berkeley, California, USA
}

A. H. Taub

Mathematics Department, University of California, Berkeley, California, USA

Received August 10, 1972

\begin{abstract}
Solutions of the Cauchy problem associated with the Einstein field equations which satisfy general initial conditions are obtained under the assumptions that (1) the source of the gravitational field is a perfect fluid with pressure, $p$, equal to energy density, $w$, and (2) the space-time admits the three parameter group of motions of the Euclidean plane, that is, the space-time is plane symmetric. The results apply to the situation where the source of the gravitational field is a massless scalar field since such a source has the same stress-energy tensor as an irrotational fluid with $p=w$. The relation between characteristic coordinates and comoving ones is discussed and used to interpret a number of special solutions. A solution involving a shock wave is discussed.
\end{abstract}

\section{Introduction}

The Einstein field equations for a self-gravitating perfect fluid with rest energy density $w$, pressure $p$ and four-velocity $u^{\mu}$ may be written as

where

$$
G^{\mu v}=R^{\mu v}-\frac{1}{2} g^{\mu v} R=-T^{\mu v}=-\left[(w+p) u^{\mu} u^{v}-p g^{\mu v}\right]
$$

$$
u^{\mu} u_{\mu}=1
$$

and the units are chosen so that the velocity of light $c=1$ and Newton's constant of gravitation $G=1 / 8 \pi$. As is well-known, Eqs. (1.1) must be supplemented by an additional requirement on the motion of the fluid. This may be taken to be an equation of state of the form

$$
p=p(w) .
$$

The velocity of sound in the fluid is then given by

$$
a^{2}=\frac{d p}{d w} .
$$

^ Present address: Departamento de Fisica, Universidad de Chile, Santiago, Chile. 
Since we must have $a \leqq 1$, it is evident that a limiting form of the equation of state is

$$
p=w .
$$

We shall show below that if the fluid satisfies this equation of state and if in addition its motion is irrotational, that is if

$$
u_{\mu}=\phi_{, \mu} / \sqrt{g^{\sigma \tau} \phi_{, \sigma} \phi_{, \tau}}=\phi_{, \mu} / \sqrt{\phi_{, \sigma} \phi^{, \sigma}}
$$

then Eqs. (1.1) may be written as

$$
G_{\mu \nu}=-2\left[\phi_{, \mu} \phi_{, v}-\frac{1}{2}\left(\phi_{, \sigma} \phi^{, \sigma}\right) g_{\mu \nu}\right],
$$

the Einstein field equations for the gravitational field due to the scalar field $\phi$. Eqs. (1.7) may also be written as

where $\sigma=\sqrt{2} \phi$.

$$
R_{\mu \nu}=-\sigma_{, \mu} \sigma_{, v}
$$

These equations are simply related to the Brans-Dicke field equations in vacuum. The latter equations may be reduced to

by defining

$$
R_{\mu \nu}=\sigma_{, \mu} \sigma_{, v}
$$

and

$$
\begin{gathered}
e^{\sigma / \sqrt{\omega+3 / 2}}={ }_{B-D} \phi \\
g_{\mu \nu}=e^{\sigma / \sqrt{\omega+3 / 2}}{ }_{B-D} g_{\mu \nu}
\end{gathered}
$$

where ${ }_{B-D} \phi$ and ${ }_{B-D} g_{\mu v}$ are the quantities occurring in the Brans-Dicke theory [1]. Thus, these three theories are all simply related.

The derivation of Eq. (1.7) from Eqs. (1.1), (1.5) and (1.6) is as follows. It is a consequence of the Bianchi identities that

and that

$$
u^{v} w_{, v}=-(p+w) u_{; v}^{v}
$$

where

$$
\begin{aligned}
h_{\mu}^{v} p_{, v} & =(p+w) u^{v} u_{\mu ; v} \\
h_{\mu}^{v} & =g_{\mu}^{v}-u^{v} u_{\mu} .
\end{aligned}
$$

If Eq. (1.3) obtains we may define a function

$$
\Sigma(w)=\int \frac{d p}{p+w}
$$

Eq. (1.9) may be then written as

$$
\left[e^{-\Sigma}(p+w) u^{\mu}\right]_{; \mu}=0 .
$$

It follows from Eqs. (1.6) and (1.11) that Eqs. (1.10) become

$$
\left[\Sigma-\ln \sqrt{\phi_{, \lambda} \phi^{, \lambda}}\right]_{, \mu}=\phi_{, \mu}\left[\frac{\phi^{\lambda}, \Sigma_{\lambda}}{\phi_{, \lambda} \phi^{, \lambda}}-\frac{\phi^{, \alpha} \phi^{, \beta} \phi_{; \alpha \beta}}{\left(\phi_{, \lambda} \phi^{, \lambda}\right)^{2}}\right] .
$$


Hence $\Sigma-\ln \sqrt{\phi,{ }_{\lambda} \phi^{, \lambda}}$ is a function of $\phi$ alone. Now we may always replace $\phi$ by $\bar{\phi}=F(\phi)$ and not change $u_{\mu}$. Hence it is no restriction to require that

$$
\Sigma(w)=\ln \sqrt{\phi_{, \lambda} \phi^{, \lambda}} .
$$

This equation determines $w$ and hence $p$ as a function of the scalar $\phi_{, \lambda} \phi^{, \lambda}$. Further, it is equivalent to Eqs. (1.10) when Eqs. (1.3) and (1.6) obtain.

Eq. (1.12) may now be written as

When $p=w$,

and Eq. (1.14) becomes

$$
\left(e^{-2 \Sigma}(p+w) \phi_{, \mu} g^{\mu v} \sqrt{-g}\right)_{, v}=0 .
$$

$$
p=w=\frac{1}{2} \sigma_{, \lambda} \sigma^{, \lambda}
$$

$$
\square \sigma=\left(\sigma_{, \mu} g^{\mu \nu} \sqrt{-g}\right)_{, v}=0,
$$

the wave equation for the scalar field $\phi$, and Eqs. (1.1) become Eqs. (1.7) or equivalently Eqs. (1.8).

It is the purpose of this paper to discuss the solutions of Eqs. (1.8) under the additional assumption that the space-time admits the threeparameter group of the Euclidean plane, i.e., it is plane symmetric. This assumption enables one to reduce the problem to one of solving a simple linear equation for the function $\sigma$. The discussion given below will make use of two coordinate systems; a coordinate system based on the characteristics of this equation and one in which the fluid is at rest, a comoving coordinate system. The relation between these coordinates will be discussed. We shall also discuss the Riemann function for the equation satisfied by $\sigma$ and give a formula for the solution of the Cauchy problem for the metric tensor as well as for the function $\sigma$. A number of specific examples will be treated in detail.

\section{Plane Symmetric Solution}

In this section we give a general solution of Eqs. (1.8) restricted to plane symmetry. We adopt the definition and coordinates given by Taub [2].

The line element can be written in the form

$$
d s^{2}=e^{\omega}\left(d t^{2}-d z^{2}\right)-e^{\mu}\left(d x^{2}+d y^{2}\right)
$$

where $\omega$ and $\mu$ are functions of $t$ and $z$.

It is somewhat convenient to introduce the characteristic coordinates $u=t-z$ and $v=t+z$ to get

$$
d s^{2}=e^{\omega} d u d v-e^{\mu}\left(d x^{2}+d y^{2}\right) .
$$

Differentiation with respect to $u$ and $v$ will be denoted by the subscripts + and - , respectively. 
It is a matter of direct computation to write the field equations

$$
\begin{aligned}
-\sigma_{+}^{2} & =\mu_{++}+\frac{1}{2} \mu_{+}^{2}-\mu_{+} \omega_{+}, \\
-\sigma_{-}^{2} & =\mu_{--}+\frac{1}{2} \mu_{-}^{2}-\mu_{-} \omega_{-}, \\
-\sigma_{+} \sigma_{-} & =\mu_{+-}+\frac{1}{2} \mu_{+} \mu_{-}+\omega_{+-} \\
0 & =\left(e^{\mu}\right)_{+-}, \\
\sigma_{+-} & =-\frac{1}{2}\left(\sigma_{+} \mu_{-}+\sigma_{-} \mu_{+}\right) .
\end{aligned}
$$

The equation not enumerated follows from the others. Eq. (2.5) corresponds to $\square \sigma=0$.

The nice feature of plane symmetry is that (2.4) has a general solution, namely

$$
e^{\mu}=f(u)+g(v) .
$$

If either $f$ or $g$ is a constant, then from (2.2) or (2.3) we see that $p=w=0$. Therefore this case would correspond to a plane symmetric vacuum; the solution is known [2] to be

$$
d s^{2}=\frac{d t^{2}-d z^{2}}{\sqrt{1+k z}}-(1+k z)\left(d x^{2}+d y^{2}\right) .
$$

Therefore, let us assume neither $f$ nor $g$ to be locally constant. We can then set $f=u / 2$ and $g=v / 2$ by an appropriate coordinate transformation. In these coordinates (2.5) is a linear equation for $\sigma$, namely

$$
\sigma_{+-}=-\frac{1}{2} \frac{\sigma_{+}+\sigma_{-}}{u+v} .
$$

We pick a solution of (2.7) and then solve for $\omega$ using (2.2) and (2.3). It is rewarding to see that the integrability condition for the existence of $\omega$ is just Eq. (2.7).

It is now straightforward to verify that

$$
e^{\omega}= \pm \frac{\exp \left[\int(u+v)\left(\sigma_{+}^{2} d u+\sigma_{-}^{2} d v\right)\right]}{\sqrt{u+v}}
$$

is the solution. The line integral above starts from any fixed point in the $(u, v)$ plane.

It is convenient to gather the results in the $(t, z)$ coordinates defined in $(2.1)$

$$
\begin{aligned}
\sigma_{z z} & =\sigma_{t t}+\frac{1}{t} \sigma_{t}, \\
d s^{2} & =\frac{e^{\Omega}}{\sqrt{t}}\left(d t^{2}-d z^{2}\right)-t\left(d x^{2}+d y^{2}\right), \\
\Omega & =\int t\left[\left(\sigma_{t}^{2}+\sigma_{z}^{2}\right) d t+2 \sigma_{t} \sigma_{z} d z\right] \\
p & =w=\frac{1}{2} \sqrt{t}\left(\sigma_{t}^{2}-\sigma_{z}^{2}\right) e^{-\Omega} .
\end{aligned}
$$


The conclusion is that for any solution of the linear Eq. (2.9) it is possible to generate a solution of the field equations $R_{\mu v}=-\sigma_{, \mu} \sigma_{, v}$.

We notice that if $\left(g_{\mu \nu}, \sigma\right)$ is any solution then $\left(\lambda g_{\mu \nu}, \sigma\right)$ is also a solution, whenever $\lambda$ is a constant. From now on it shall be understood that all line elements can be multiplied by a constant conformal factor.

\section{Solution in Comoving Coordinates}

In this section we shall discuss the same problem of the preceding one in comoving coordinates. Comoving coordinates are usually adopted in hydrodynamics and therefore it is of interest to see what difficulties arise.

We shall start from the following line element

$$
d s^{2}=e^{2 \phi} d T^{2}-e^{2 \psi} d Z^{2}-R^{2}\left(d X^{2}+d Y^{2}\right) .
$$

When the pressure is non-vanishing the field equations can be written in the form [3]

$$
\begin{aligned}
2 m & =R\left(e^{-2 \phi} R_{T}^{2}-e^{-2 \psi} R_{Z}^{2}\right), \\
m_{Z} & =w R^{2} R_{Z}, \\
m_{T} & =-p R^{2} R_{T}, \\
0 & =R_{Z T}-\phi_{Z} R_{T}-\psi_{T} R_{Z}, \\
\phi_{Z} & =-\frac{P_{Z}}{w+p}, \\
\psi_{T} & =-\frac{w_{T}}{w+p}-\frac{2 R_{T}}{R} .
\end{aligned}
$$

With the assumption $p=w$ the solution of the Eqs. (3.6) and (3.7) is

$$
e^{-\phi}=\sqrt{p} \quad e^{-\psi}=\sqrt{p} R^{2}
$$

where functions of integration have been absorbed in the definition of $T$ and $Z$. The line element (3.1) now reads

$$
d s^{2}=\frac{1}{p}\left(d T^{2}-\frac{d Z^{2}}{R^{2}}\right)-R^{2}\left(d X^{2}+d Y^{2}\right) .
$$

It is possible to get a single equation for $R$ alone from the field equations:

$$
\left(R^{2} R^{2}\right)_{Z}=\left(\frac{R^{2} T}{R^{2}}\right)_{T}
$$


Combining (3.2), (3.3), (3.4) and (3.8) we get

and

$$
\begin{aligned}
\frac{m_{T}}{m} & =-\frac{2 R R_{T}}{R_{T}^{2}-R^{4} R^{2}{ }_{Z}} \\
\frac{m_{Z}}{m} & =\frac{2 R R_{Z}}{R_{T}^{2}-R^{4} R_{Z}^{2}}
\end{aligned}
$$

$$
p=w=\frac{2 m}{R\left(R_{T}^{2}-R^{4} R_{Z}^{2}\right)} .
$$

So a solution of the Eq. (3.9) generates a solution of the field equations.

It is interesting that the non-linear partial differential Eq. (3.9) can be solved completely.

It is convenient to work with $R^{2}$ instead of $R$ and so we shall define

$$
t=R^{2}
$$

so $t$ satisfies

$$
\left(t t_{Z}\right)_{Z}=\left(\frac{t_{T}}{t}\right)_{T}
$$

Eq. (3.11) assures us for the existence of a function $z(T, Z)$ such that

$$
t t_{Z}=z_{T} \quad \frac{t_{T}}{t}=z_{Z}
$$

or

$$
z_{T}-t t_{Z}=0 \quad t_{T}-t z_{Z}=0 .
$$

A well-known method to solve these equations is to reverse the role of dependent and independent variables to obtain a linear system. That is, instead of looking for $t=t(T, Z)$ and $z=z(T, Z)$ we solve for $T=T(t, z)$ and $Z=Z(t, z)$.

It will now be shown that this inversion is possible whenever the density does not vanish. We shall therefore show that when the Jacobian $J=t_{T} z_{Z}-t_{Z} z_{T}$ vanishes so does the density.

Let us take a solution of (3.12) with $J=0$ and at the same time assume the corresponding density to be non-zero. This will lead us to a contradiction. The advantage of this method is that we can use Eqs. (3.2)-(3.7). Combining (3.2), (3.8) and (3.10) we get $m=0$. From (3.3) and (3.4) we get that $R$ is a constant function. Now from (3.6) and (3.7) we learn that $\phi=\psi$, so we are again returned to the line element $(2.1)$ with $e^{\mu}=R^{2}$ constant. Field Eqs. (2.2) and (2.4) then tell us that the solution is a flat space-time which cannot have but zero density. This is the sought contradiction. 
With the help of inversion formulas

(3.12) reads

$$
\begin{aligned}
t_{Z} & =-J T_{z} \quad z_{Z}=J T_{t} \\
t_{T} & =J Z_{z} \quad z_{T}=-J Z_{t} \\
J & =t_{T} z_{Z}-t_{Z} z_{T}
\end{aligned}
$$

$$
Z_{t}-t T_{z}=0 \quad Z_{z}-t T_{t}=0
$$

which are linear equations.

The scheme for solving (3.18) is to pick any solution of

$$
T_{z z}=T_{t t}+\frac{1}{t} T_{t}
$$

and to calculate $Z=Z(t, z)$ from

$$
d Z=t\left(T_{z} d t+T_{t} d z\right)
$$

which involves a single quadrature. In the next section we shall give a procedure to avoid this step.

All that is left now is to find $p$. For this, we use the formulas

derived from (3.2).

$$
p=\frac{8 m}{J \sqrt{t}} \quad J=\left[t\left(T_{t}^{2}-T_{z}^{2}\right)\right]^{-1}
$$

We can now calculate $m$ from

$$
\begin{aligned}
\frac{m_{t}}{m} & =-4 t\left(T_{t}^{2}+T_{z}^{2}\right), \\
\frac{m_{z}}{m} & =-8 t T_{t} T_{z} .
\end{aligned}
$$

This last set of equations are derived in a straight-forward manner from (3.2), (3.3), and (3.4).

To summarize, we define $\Omega$ by

$$
d \Omega=4 t\left[\left(T_{t}^{2}+T_{z}^{2}\right) d t+2 T_{t} T_{z} d z\right],
$$

and the line element then becomes

$$
d s^{2}=\frac{e^{\Omega}}{8 \sqrt{t}\left(T_{t}^{2}-T_{z}^{2}\right)}\left[d T^{2}-\frac{d Z^{2}}{t^{2}}\right]-t\left(d X^{2}+d Y^{2}\right) .
$$

The correspondence of this line element to $(2.10)$ is seen by defining $\sigma=2 T$ and adding a constant to $\Omega$.

We shall now give a prescription to transform any solution in characteristic coordinates to comoving ones for the purpose of interpretation. 
Obviously $\sigma$ can be chosen to be the comoving time. It can also be easily seen that the coordinate $Z$ defined by

$$
d Z=t\left(\sigma_{z} d t+\sigma_{t} d_{z}\right)
$$

is orthogonal to $\sigma, x$ and $y$ and therefore $Z$ is comoving. Eq. (2.9) guarantees that the differential defining $Z$ is exact.

We now have the required transformation formulas

$$
\begin{aligned}
& T=\sigma(t, z) \\
& Z=Z(t, z) \\
& X=x \\
& Y=y
\end{aligned}
$$

where $T, Z, X$ and $Y$ are the comoving coordinates. All that is left to verify is the existence of the inverse transformation. The Jacobian of (3.2) is

$$
\frac{\partial(T, Z, X, Y)}{\partial(t z x y)}=t\left(\sigma_{t}^{2}-\sigma_{z}^{2}\right)
$$

which can be seen from (2.11) to vanish only when $p=w=0$ or on the surface $t=0$. In any event, the surface $t=0$ is, from (2.10), pathological and in all examples studied so far it is singular.

We now write the line element (2.10) in comoving coordinates to obtain once again (3.18).

\section{Cauchy Problem}

We have by now reduced the problem of integrating the field equations to that of solving the simple linear differential Eq. (2.7), namely

$$
\sigma_{+-}=-\frac{1}{2} \frac{\sigma_{+}+\sigma_{-}}{u+v} .
$$

This equation has many simple solutions, some of which we shall discuss in the next section. Here we shall give a method of generating solutions from given physical data. The Riemann-Volterra representation for linear hyperbolic equations fits our needs very nicely. This method will give us a solution of (4.1) for prescribed values of $\sigma$ and its derivatives along an arbitrary curve in the $(u, v)$ plane. Clearly, any one derivative of $\sigma$ not tangent to the curve is sufficient. We shall give a physical interpretation for this kind of data. 


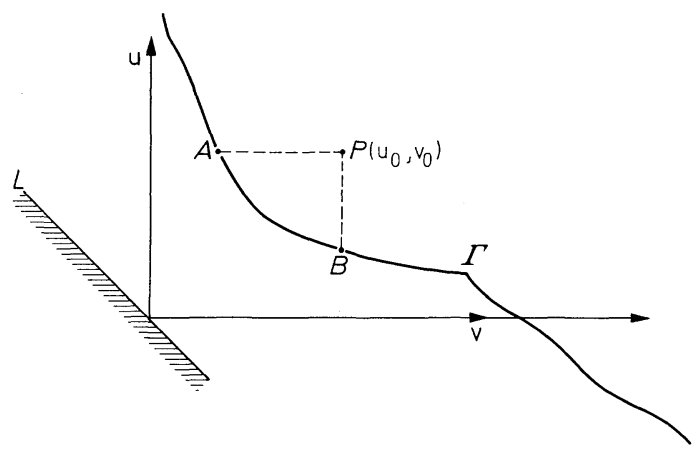

Fig. 1. When data on $\Gamma$ is given, $\sigma(P)$ is determined. $L$ is the curve $u+v=t=0$

Now let us refer to Fig. 1. The value of $\sigma$ at any point $p=\left(u_{0}, v_{0}\right)$ is given by the Riemann-Volterra representation [4].

$$
\begin{aligned}
\sigma(P)= & \frac{1}{2}[\sigma(A) R(A)+\sigma(B) R(B)] \\
& -\frac{1}{2} \int_{A B} R\left[\sigma_{+} d u-\sigma_{-} d v\right] \\
& -\frac{1}{2} \int_{A B} \sigma\left[\left(\frac{R}{u+v}-R_{+}\right) d u-\left(\frac{R}{u+v}-R_{-}\right) d v\right]
\end{aligned}
$$

where $R$ is the Riemann function. Now $R$ is uniquely determined by the differential Eq. (4.1) and is, fortunately, known to be

$$
R\left(u v ; u_{0} v_{0}\right)=\sqrt{\frac{u+v}{u_{0}+v_{0}}} F\left(\frac{1}{2}, \frac{1}{2}, 1 ;-\frac{\left(u-u_{0}\right)\left(v-v_{0}\right)}{(u+v)\left(u_{0}+v_{0}\right)}\right)
$$

where $F$ is a hypergoemetric function. We might add that $u_{0}$ and $v_{0}$ play the role of parameters in (4.2).

We shall now use formula (4.2) in the following way: pick any spacelike curve $\Gamma$ in the $(u, v)$ plane and let it correspond to an equal comoving time surface; that is, $\sigma=0$ on $\Gamma$. Specification of the density on $\Gamma$ is enough to generate a solution, as we shall see in a moment.

Under these conditions (4.2) reads

$$
\sigma(P)=-\frac{1}{2} \int_{A B} R\left[\sigma_{+} d u-\sigma_{-} d v\right] .
$$

Now let $u=u(\gamma)$ and $v=v(\gamma)$ be the parametric equations of $\Gamma$. Since $\sigma=0$ on $\Gamma$ we shall have

$$
\sigma_{-}(\gamma)=-\sigma_{+}(\gamma) \frac{d u / d \gamma}{d v / d \gamma}
$$


and from here

follows.

$$
\sigma\left(u_{0}, v_{0}\right)=-\int_{A B} R\left[u(\gamma), v(\gamma) ; u_{0} v_{0}\right] \sigma_{+}(\gamma) \frac{d u}{d \gamma} d \gamma .
$$

All that is now required is to express $\sigma_{+}(\gamma)$ in terms of the density. For this we use (1.14), (2.8) and (4.5). We then get the following expression for $w(\gamma)$

where

$$
w(\gamma)=\frac{1}{2}(u+v)^{\frac{1}{2}} e^{-\Omega} \frac{-d u / d \gamma}{d v / d \gamma} \sigma_{+}^{2}(\gamma)
$$

$$
\Omega=\int_{\gamma_{0}}^{\gamma}(u+v) \frac{d u}{d \gamma}\left(1+\left(\frac{d u / d \gamma}{d v / d \gamma}\right)\right) \sigma_{+}^{2}(\gamma) d \gamma
$$

and $\gamma_{0}$ is defined to be the value of the parameter $\gamma$ for which $\Omega$ vanishes.

Our problem now is to invert (4.7), that is, to express $\sigma_{+}(\gamma)$ in terms of $w(\gamma)$. The inversion is accomplished by rewriting (4.7) in the following way:

$$
(u+v)^{\frac{1}{2}} \frac{d(u+v)}{d \gamma} w=\frac{1}{2} \frac{d}{d \gamma} e^{-\Omega} .
$$

Integrating (4.9) and differentiating the result with respect to $\gamma$ then gives us the desired result

$$
\sigma_{+}^{2}(\gamma)=-\frac{2}{\sqrt{u+v}} \frac{d v / d \gamma}{d u / d \gamma} \frac{w(\gamma)}{1+\frac{4}{3} \int_{\gamma_{0}}^{\gamma} \frac{d(u+v)^{3 / 2}}{d \gamma} w(\gamma) d \gamma} .
$$

Formula (4.6) combined with (4.10) can be used to study the behavior of matter for arbitrary times. We have done some explicit integrations of (4.6) for simple initial matter distributions and obtained solutions that could be derived by more elementary means, these special cases are discussed in the next section.

We can derive the following property from (4.6): if matter is initially confined to one side of an infinite plane, $\sigma_{\lambda} \sigma^{\lambda}$ will eventually become negative.

The theorem as it stands is not true unless we add some remarks. First, let us point out that the sign in front of $e^{\Omega}$ in formula (2.10) is arbitrary. This means that our $t$ coordinate can either be time-like or space-like. We notice that the line element has a singularity at $t=0$. Therefore if we choose $t$ to be a time coordinate the singularity is of the Big Bang type, but if $t$ is a space coordinate we get a Big Wall type singularity. The theorem is valid if we exclude this last kind of singularity; that is, if we choose $t$ to be a time coordinate. 
We now proceed to prove the statement. Let $\sigma$ vanish on the curve $\Gamma$ in Fig. 1. Let $w$ be zero on $\Gamma$ for $u \leqq 0$. This situation can always be accomplished because the whole theory is invariant under the following transformations:

$$
\begin{aligned}
u^{\prime} & =u+\Delta \\
v^{\prime} & =v-\Delta .
\end{aligned}
$$

It is understood that $u_{0}$ and $v_{0}$ transform in the same manner. Let us also assume that $\Gamma$ is at a finite distance away from the Big Bang $u+v=0$ and $u+v$ evaluated on $\Gamma$ has a nonvanished minimum. We shall evaluate

$\sigma\left(u_{0}, v_{0}\right)$ in a region satisfying the conditions $u_{0} \ll u+v$ and $v_{0} \gg u+v$, where the values of $u+v$ range over that portion of $\Gamma$ for which the density is non-zero and $u_{0} \leqq \bar{u}>0$ for some $\bar{u}$. Under these conditions the argument of the hypergoemetric function (4.3) is as close to zero as we please. If in addition we choose the parameter $\gamma$ to be $u$ itself, we get the following asymptotic expression for $\sigma\left(u_{0}, v_{0}\right)$ :

$$
\sigma\left(u_{0}, v_{0}\right) \simeq \frac{1}{\sqrt{v_{0}}} \int_{0}^{u_{0}} \sqrt{u+v(u)} \sigma_{+}(u) d u .
$$

In other words, $\sigma\left(u_{0}, v_{0}\right)$ has the form

$$
\sigma\left(u_{0}, v_{0}\right) \simeq \frac{E\left(u_{0}\right)}{\sqrt{v_{0}}} .
$$

Evaluation of $\sigma_{\lambda} \sigma^{\lambda}$ from this expression gives

$$
\sigma_{\lambda} \sigma^{\lambda} \simeq-\frac{1}{4} e^{-\omega} v_{0}^{-2} \frac{d\left(E^{2}\right)}{d u_{0}} .
$$

We therefore see that it is necessarily negative for small values of $u_{0}$ since $E(0)=0$ and $E^{2}$ is positive.

We would like to add that if matter is not orginally confined, $\sigma_{\lambda} \sigma^{\lambda}$ need not grow negative.

A problem of physical interpretation arises when $\sigma_{\lambda} \sigma^{\lambda}$ is negative. First of all we see from (1.6) and from the fact that $\sigma=\sqrt{2} \phi$, that $u_{\mu}$ is imaginary. Since we are primarily looking for solutions of (1.1) it is clear that when $\sigma_{\lambda} \sigma^{\lambda}$ is negative we still have a solution of (1.1) if

$$
\begin{aligned}
p+w & =-\sigma_{\lambda} \sigma^{\lambda} \\
p & =\frac{1}{2} \sigma_{\lambda} \sigma^{\lambda} \\
u_{\mu} & =i \frac{\sigma, \mu}{\sqrt{\sigma_{\lambda} \sigma^{\lambda}}} .
\end{aligned}
$$


This formal solution has to be ruled out on physical grounds because $u_{\mu}$ in (4.14) is space-like. Two attitudes can now be adopted: either we eliminate the region of space-time where $\sigma_{\lambda} \sigma^{\lambda}$ is negative or we fill the region with some other kind of fluid. The first alternative does not seem reasonable because the line element does not show any pathologies where $\sigma_{\lambda} \sigma^{\lambda}$ becomes negative. We shall now try to find a reasonable stressenergy tensor for the line element (2.10) where $\sigma_{\lambda} \sigma^{\lambda}$ is negative ${ }^{1}$.

From (1.8) we get the following stress-energy tensor:

$$
T_{\mu v}=\sigma_{, \mu} \sigma_{, v}-\frac{1}{2} \sigma_{, \lambda} \sigma^{, \lambda} g_{\mu v}
$$

where $\sigma_{, \mu}$ is a space-like vector field. From (3.19) we learn that $Z_{, \mu}$ is orthogonal to $\sigma_{, \mu}, x_{, \mu}$ and $y_{, \mu}$ and therefore it is a time like vector field. Now let $\hat{Z}_{, \mu}, \hat{\sigma}_{, \mu}, \hat{x}_{, \mu}$ and $\hat{y}_{, \mu}$ denote the corresponding unit vector fields. Using the fact that

$$
g_{\mu \nu}=\hat{Z}_{, \mu} \hat{Z}_{, v}-\hat{\sigma}_{, \mu} \hat{\sigma}_{, v}-\hat{x}_{, \mu} \hat{x}_{, v}-\hat{y}_{, \mu} \hat{y}_{, v}
$$

the stress-energy tensor (4.15) can be written as:

$$
T_{\mu \nu}=-\frac{1}{2} \sigma_{, \lambda} \sigma^{, \lambda}\left[\hat{Z}_{, \mu} \hat{Z}_{, v}+\hat{\sigma}_{, \mu} \hat{\sigma}_{, v}-\hat{x}_{, \mu} \hat{x}_{, v}-\hat{y}_{, \mu} \hat{y}_{, v}\right] .
$$

The stress- energy tensor is that of an anisotropic fluid with positive rest energy density $-\frac{1}{2} \sigma_{\lambda} \sigma^{\lambda}$ and vanishing heat-flow vector. Wo would like to add that the energy inequality $T_{\mu \nu} u^{\mu} u^{\nu} \geqq 0$, for all time-like vectors $u^{\mu}$, is satisfied. The widely adopted inequality $\left(T_{\mu \nu}-\frac{1}{2} T g_{\mu \nu}\right) u^{\mu} u^{v} \geqq 0$ is satisfied too [5]. We have therefore achieved a reasonable model.

\section{Special Cases}

In this section we shall consider particular plane symmetric solutions of the perfect fluid model with $p=w$ equation of state.

For the purpose of conversion to comoving coordinates it is convenient to notice a simple manner of integrating Eq. (3.19). The procedure is to pick a solution $\Sigma(t, z)$ of Eq. (2.9) and set $\sigma=\Sigma_{z}$ which solves the same equation. It then follows from (3.19) that $Z=t \Sigma_{t}$. In this way we avoid one integration.

(a) Static Space-time. If one assumes the corresponding timelike Killing vector orthogonal to the surface $\sigma=$ constant, it follows that the static case is characterized by $Z=Z(t)$, and $\Sigma$ turns out to be given by $\Sigma=\frac{1}{2} a\left(z^{2}+\frac{1}{2} t^{2}\right)$ where a is a constant. From here we get $\Omega=a^{2} t^{2} / 2$. It is, in (2.10), necessary to choose the minus sign to get time-like fluid

\footnotetext{
1 This approach has been suggested to us by R. K. Sachs.
} 
lines. The static solution then reads

$$
\begin{aligned}
d s^{2} & =\frac{e^{a^{2} t^{2} / 2}}{\sqrt{t}}\left(d z^{2}-d t^{2}\right)-t\left(d x^{2}+d y^{2}\right) \\
p & =w=\frac{1}{2} a^{2} \sqrt{t} e^{-a^{2} t^{2} / 2} .
\end{aligned}
$$

In comoving coordinates we have

$$
\begin{aligned}
d s^{2} & =\frac{e^{a^{2} Z^{2} / 2}}{\sqrt{a Z}}\left(d T^{2}-d Z^{2}\right)-a Z\left(d x^{2}+d y^{2}\right) \\
p & =w=\frac{1}{2} a^{2} \sqrt{a Z} e^{-a^{2} Z^{2} / 2}
\end{aligned}
$$

where a minor coordinate transformation has been made.

The interpretation is as follows: we choose $d$ to be the proper distance from $Z=0$ along $x, y, T$ constant. The density vanishes at $Z=\infty$ and at $Z=0$ as described by Fig. 2 . The plane $Z=0$ behaves like a hard wall. It can be shown that all time-like geodesics oscillate between two $Z$ planes determined by initial conditions. No time-like geodesic ever touches the wall. We can, nevertheless, imagine the space-time for $Z<0$ to be the vacuum solution (2.6). Strictly speaking one doesn't know the matching condition for singular hypersurfaces but the type of divergence from both sides of $Z=0$ is exactly the same.

We conclude that the only way to have a static solution is to introduce an additional boundary condition, the wall.

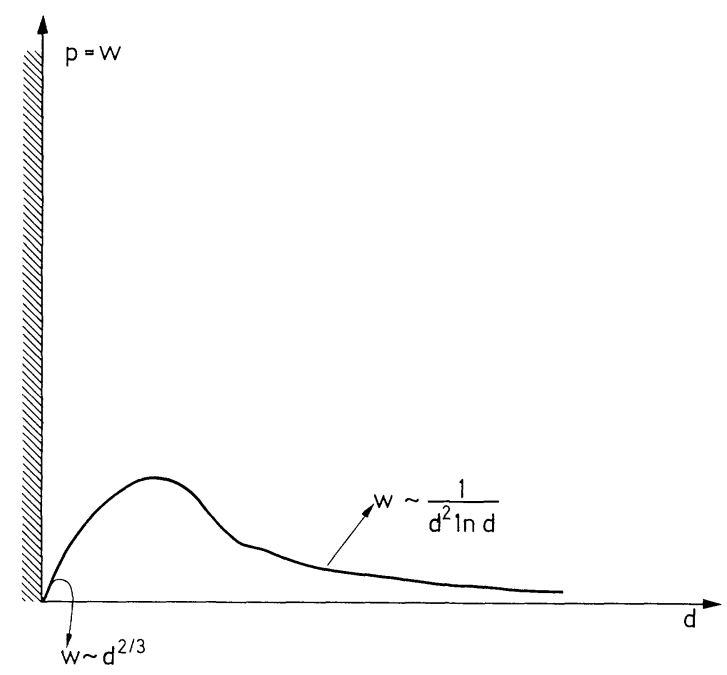

Fig. 2. Density dependence for the static space-time 
(b) Robertson-Walker Space-time. Without going through details we find $\Sigma$ to be given by

the metric by

$$
\Sigma=\sqrt{\frac{3}{2}} z \ln t
$$

$$
d s^{2}=t\left(d t^{2}-d z^{2}-d x^{2}-d y^{2}\right)
$$

and the density by

$$
p=w=\frac{3}{4} \frac{1}{t^{3}} .
$$

(c) Asymptotic Robertson-Walker Space-time. Here we shall consider a particular solution which is inhomogeneous and anisotropic but tends to an homogeneous space-time for large times. The solution depends on two parameters $\alpha$ and $\beta$. For $\alpha^{2}+\beta^{2}=3 / 2$ we get RobertsonWalker space-time asymptotically.

The case in question is defined by

$$
\sigma=\alpha \ln t+\beta \cos ^{-1} z / t \quad \alpha>\beta>0 .
$$

Without going through details we give the main functions

$$
\begin{gathered}
Z=\alpha z-\beta \sqrt{t^{2}-z^{2}} \\
\Omega=\left(\alpha^{2}+\beta^{2}\right) \ln t+\beta^{2} \ln \left(1-z^{2} / t^{2}\right)+2 \alpha \beta \cos ^{-1} z / t .
\end{gathered}
$$

The variables $z$ and $t$ are restricted to $z^{2} \leqq t^{2}$. We shall now make use of Fig. 3 to state the behavior of the fluidlines. The fluid-lines $P$ between $N$

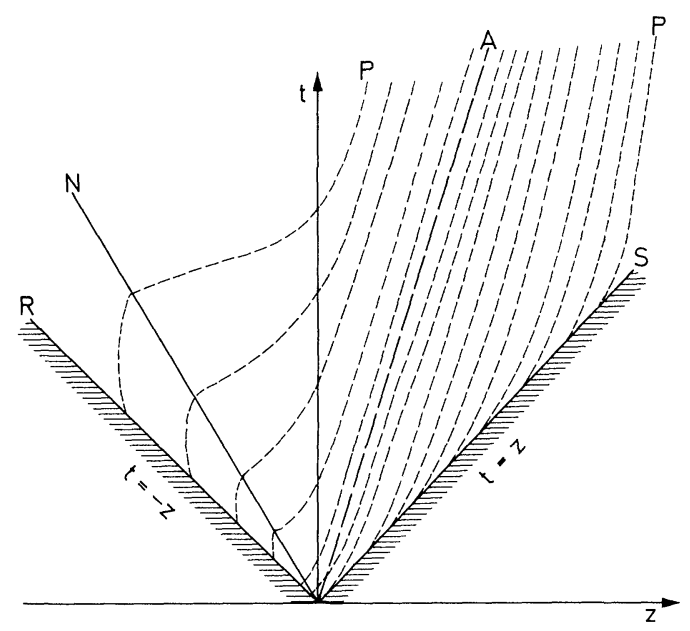

Fig. 3. Behavior of the fluid-lines for the particular solution " $d$ " 
and $S$ are hyperbolas given by

$$
\frac{t^{2}}{\left[Z / \sqrt{\alpha^{2}+\beta^{2}}\right]^{2}}-\frac{\left[z-\alpha Z /\left(\alpha^{2}+\beta^{2}\right)\right]^{2}}{\left[\beta Z / \alpha^{2}+\beta^{2}\right]^{2}}=1 .
$$

This last expression comes from the formula for $Z$ above.

In the region between $R$ and $N$ one has that $\sigma_{\lambda} \sigma^{\lambda}<0$, so we therefore make use of the discussion at the end of section 4 to see that the fluid-lines are now given by $\sigma(t, z)=$ constant. $N$ is the locus of points where $\sigma_{\lambda} \sigma^{\lambda}=0$ and is given by

$$
t=-\frac{\alpha^{2}+\beta^{2}}{\alpha^{2}-\beta^{2}} z
$$

itself a fluid line, $A$ is parallel to the hyperbola's asymptotes and is given by

$$
t=\frac{1}{\beta} \sqrt{\alpha^{2}+\beta^{2}} z \text {. }
$$

We see that the fluid world-lines approach straight lines for $t \rightarrow \infty$. We shall now show that in this limit, space-time is homogeneous, and for some values of the parameters, Robertson-Walker. All we have to do is to carry out the inversion to comoving coordinates for $t$ large and $Z$ finite. The calculation is straight-forward but rather long, so we omit it. The result in comoving coordinates is:

$$
\begin{aligned}
d s^{2}= & K T^{\left(\alpha^{2}+\beta^{\left.2-\frac{1}{2}\right)}\right.}\left[1-\frac{3}{2} \frac{\beta}{\sqrt{\alpha^{2}+\beta^{2}}} \frac{Z^{\prime}}{T}\right]\left(d T^{2}-d Z^{\prime 2}\left[1-\frac{\beta}{\sqrt{\alpha^{2}+\beta^{2}}} \frac{Z^{\prime}}{T}\right]\right) \\
& -T\left[1+\frac{\beta}{\sqrt{\alpha^{2}+\beta^{2}}} \frac{Z^{\prime}}{T}\right]\left(d x^{2}+d y^{2}\right), \\
p=w & =\frac{\alpha^{2}}{K}\left[1+\frac{3}{2} \frac{\beta}{\sqrt{\alpha^{2}+\beta^{2}}} \frac{Z^{\prime}}{T}\right] T^{-\left(\alpha^{2}+\beta^{2}+3 / 2\right)}
\end{aligned}
$$

where

$$
\begin{aligned}
K & =\left[\frac{\alpha^{2}}{\alpha^{2}+\beta^{2}}\right]^{\beta^{2}} \frac{\alpha^{2}}{\alpha^{2}+\beta^{2}} \exp \left(2 \alpha \beta \cos ^{-1} \frac{\beta}{\sqrt{\alpha^{2}+\beta^{2}}}\right), \\
T & =e^{\left(\sigma-\beta \cos ^{-1} \beta / \sqrt{\alpha^{2}+\beta^{2}} / \alpha\right.}, \\
Z^{\prime} & =Z / \alpha .
\end{aligned}
$$

Therefore we get that for any group of fluid world-lines, defined in some interval $Z_{1}^{\prime} \leqq Z^{\prime} \leqq Z_{2}^{\prime}$ and times $T \gg\left(\beta / \sqrt{\alpha^{2}+\beta^{2}}\right) Z_{2}^{\prime}$ the space-time is homogeneous. We also see that only $\alpha^{2}+\beta^{2}=3 / 2$ corresponds to a Robertson-Walker space-time. 
(d) Shocks. By shocks we understand a three-surface across which the pressure is discontinuous. We shall show that the present theory does admit shock solutions by means of a special example. We shall also sketch a method to generate arbitrary shocks solutions. The solution to the continuity conditions across the shock has been given by Taub [6]. From these continuity conditions it can be shown that the shock can occur only on characteristic surfaces, that is, along $u=$ constant or $v=$ constant. These conditions in our case just amount to the continuity of $e^{-\Omega}$ defined by (2.10), and the derivatives of $\sigma(u, v)$ within the shock surface.

Let us assume the shock at $u=0$. One way to construct these spacetimes is to first assume a background solution $\sigma_{B}(u, v)$ with no discontinuities. We then superimpose another solution $\sigma_{S}(u, v)$ defined only for $u<0$ such that $\sigma_{S}(0, v)=0$. In this way we insure the continuity of $\sigma_{-}$ across the shock. A sufficient condition for the continuity of $e^{-\Omega}$ is that $\sigma_{+}$be finite on both sides of the shock. Fortunately, it is simple to give an integral representation for $\sigma_{S}(u, v)$. First we notice that $G(x) / \sqrt{(x-u)(v+x)}$ is a solution of $(2.7)$ for arbitrary $x$ and then we make a suitable superposition, namely,

$$
\sigma_{S}(u, v)=\int_{u}^{0} \frac{G(x) d x}{\sqrt{(x-u)(v+x)}} \quad u \leqq 0 .
$$

This integral solves (2.7) even with the variable limit of integration if $G(x)$ satisfies $\lim _{x \rightarrow 0} x G(x)=0$. We prove the statement by assuming the usual rules of differentiation for parametric integrals after making the change of variable to $\theta=x / u$. Using the same method we can see that $\sigma_{S}(0, v)=0$ follows from $\lim _{x \rightarrow 0} \sqrt{-x} G(x)=0$. The jump pressure can only be due to the jump in $\sigma_{+}(u, v)$ at $u=0$. The condition for a discontinuity in $\sigma_{+}(u, v)$ is just $\lim _{x \rightarrow 0} \frac{d}{d x} \sqrt{-x} G(x) \neq 0$. The simplest choice for $G(x)$ is

$$
G(x)=-\frac{\gamma}{2} \sqrt{-x}
$$

where $\gamma$ is an arbitrary constant.

To give just one example, let us choose

$$
\begin{aligned}
\sigma_{B} & =\sqrt{\frac{3}{2}} \ln (u+v) \\
\sigma_{S} & =-\gamma \sqrt{v} \int_{0}^{\pi / 2} \frac{(-u / v) \sin ^{2} \alpha d \alpha}{\sqrt{1+u / v \sin ^{2} \alpha}}
\end{aligned}
$$

where $\sigma_{B}$ corresponds to the Robertson-Walker space-time and $\sigma_{S}$ comes from our simple choice of $G(x)$ [7]. It is straightforward to get 
the jump in pressure; the result is

$$
\frac{\Delta p}{p}=\sqrt{\frac{2}{3}} \frac{\pi}{4} \gamma v^{\frac{1}{2}}
$$

where $p$ stands for the pressure at $u=0$ in the Robertson-Walker model. For positive $\gamma$ the pressure on the Robertson-Walker side is the lowest.

We have checked the continuity of $e^{-\Omega}$. The fluid lines are everywhere time-like for positive $\gamma$. The formula for the jump in pressure is much more general. It is simple to see that for small negatives value of $u$

$$
H \sigma_{S} \simeq \frac{E(u)}{\sqrt{v}} \quad \text { where } \quad E(u)=\int_{u}^{0} \frac{G(x) d x}{\sqrt{x-u}}
$$

and from here it then follows that

$$
\frac{\Delta p}{p}=\sqrt{\frac{2}{3}} E_{+}(0) v^{1 / 2} \text {. }
$$

\section{References}

1. Dicke, R.H.: Mach's principle and invariance under transformation of units. Phys. Rev. 125, 2163 (1962).

2. Taub, A.H.: Isentropic hydrodynamics in plane symmetric space-times. Phys. Rev. 103, 454 (1956).

3. Taub, A.H.: Plane symmetric similarity solutions for self-gravitating fluids. General relativity (Papers in honor of J. L. Synge; (Ed.) L. O'Raifeartaigh) Oxford: Claredon Press 1972.

4. Courant, R., Hilbert, D.: Methods of mathematical physics, Vol. II, p. 449. New York: Interscience Publ. 1966.

5. Hawking, S. W., Penrose, R.: The singularities of gravitational collapse and cosmology. Proc. Roy. Soc. A 314, 529 (1970).

6. Taub, A.H.: Continuity conditions across null shocks. Commun. math. Phys. to appear.

7. Gradshteyn, I. S., Ryzhik, I. M.: Table of integrals, series and products, p. 233. New York: Academic Press, 1965.

Romualdo Tabensky

Physics Department

University of California

Berkeley, California 94720

USA

\author{
A. H. Taub \\ Mathematics Department \\ University of California \\ Berkeley 4, California 94720, USA
}


